\title{
New aspects of plasma sheet dynamics - MHD and kinetic theory
}

\author{
H. Wiechen \\ Institut für Astronomie und Astrophysik, Ludwig-Maximilians-Universität München, Scheinerstr. 1, D-81679 Munich, Germany
}

Received: 16 July 1998 / Revised: 18 November 1998 / Accepted 24 November 1998

\begin{abstract}
Magnetic reconnection is a process of fundamental importance for the dynamics of the Earth's plasma sheet. In this context, the development of thin current sheets in the near-Earth plasma sheet is a topic of special interest because they could be a possible cause of microscopic fluctuations acting as collective non-idealness from a macroscopic point of view. Simulations of the near-Earth plasma sheet including boundary perturbations due to localized inflow through the northern (or southern) plasma sheet boundary show developing thin current sheets in the near-Earth plasma sheet about 8-10 $R_{E}$ tailwards of the Earth. This location is largely independent from the localization of the perturbation. The second part of the paper deals with the problem of the macroscopic non-ideal consequences of microscopic fluctuations. A new model is presented that allows the quantitative calculation of macroscopic non-idealness without considering details of microscopic instabilities or turbulence. This model is only based on the assumption of a strongly fluctuating, mixing dynamics on microscopic scales in phase space. The result of this approach is an expression for anomalous non-idealness formally similar to the Krook resistivity but now describing the macroscopic consequences of collective microscopic fluctuations, not of collisions.
\end{abstract}

Key words. Magnetospheric physics (plasma sheet). Space plasma physics (kinetic and MHD theory; magnetic reconnection)

\section{Introduction}

Magnetic reconnection is widely accepted to play a crucial role in context with magnetospheric activity.

Correspondence to: hew@usm.uni-muenchen.de
This refers to the formation of plasmoids and their tailward ejection as a typical feature for almost all large isolated substorms (Moldwin and Hughes, 1993). Besides, in the near-Earth plasma sheet where the Earth's dipole field is of relevance, magnetic reconnection has to be considered as a process of relevance with respect to phenomena like pseudo-breakups (e.g. Wiechen et al. 1996).

Magnetic reconnection demands for some at least local non-idealness because of magnetic line conservation in an ideal plasma. However, in general, the magnetospheric plasma has to be considered as highly collisionfree (from a kinetic point of view) or as highly ideal (in the framework of magnetohydrodynamics) with a typical resistivity of the order of $10^{-17} \mathrm{~s}$ (Lui et al., 1993). Thus there is the question of the origin of some sufficient local non-idealness.

The solutions to this problem are microscopic instabilities or turbulences providing anomalous nonidealness on macroscopic scales (e.g. Huba et al., 1977; Papadopoulos, 1979). There are several microscopic processes discussed in the literature which may yield non-idealness on macroscopic scales, e.g. non-adiabatic particles (Schindler, 1974), lower hybrid drift instabilities (Huba et al., 1978), plasma-wave interactions (Haerendel, 1978) or chaotic particles (Büchner and Zelenyi, 1989).

Processes of special interest in this context are current-driven (micro-) instabilities like the cross-tail instability (Lui et al., 1991). For current-driven turbulence to be sufficiently effective, the local length scales of the current sheet have to be sufficiently small (i.e. of the order of the ion gyro-radius, Schindler, 1994). Those thin current sheets, indeed, are observed especially in the near-Earth plasma sheet (e.g. Sergeev et al., 1990; Pulkkinen et al., 1992). Thus, the formation of local thin current sheets is a problem of special interest with respect to the onset of magnetic reconnection.

Thin current sheets can be explained as the result of boundary perturbations in ideal MHD systems (Parker, 1972; Hahm and Kulsrud, 1982). Schindler and Birn 
(1993) have shown that dissipation-free perturbations at the, as the earthward one interpreted, boundary of a 1dimensional ideal Harris sheet yield thin current sheets near to that boundary. This line has been followed further by Wiegelmannn and Schindler (1995) considering 2dimensional tail configurations showing that thin current sheets in the central plasma sheet can result from flux transfer through the magnetopause. Rastätter et al. (1994) found current sheets in equilibrium configurations which result from the relaxation of X-point configurations after pressure perturbations. MHD-simulations of ideal 1- and 2-dimensional tail cofigurations confirm the development of thin current sheets due to dissipation-free boundary perturbations (Voge et al., 1994).

In the first part of the present paper we study the problem of the formation of thin current sheets in the near-Earth plasma sheet where the dipole field yields a significant contribution. First 2-dimensional ideal MHD-simulations (Wiechen et al., 1997) have shown, that thin current sheets can occur in the near-Earth plasma sheet due to local inflow through the plasma sheet boundary. In the present paper we show results of 3-dimensional ideal MHD-simulations starting with a model for the near-Earth plasma sheet including the Earth's dipole field, explicitly. These 3-dimensional simulations confirm the results of the previous 2dimensional simulations showing developing thin current sheets as a consequence of dissipation-free local inflow through the plasma sheet boundary even in an ideal system with more degrees of freedom to react on the perturbation.

A point of special interest in context with the formation of thin current sheets is that of their localization. We study this problem by the help of 2dimensional simulations of the near-Earth plasma sheet and show that the location of developing thin current sheets comes out to be widely independent from the localization of inflow through the plasma sheet boundary. This location of about $8-10 R_{E}$ tailwards of the Earth found in our simulations is in good agreement with the observed location of near-Earth thin current sheets during late growth phase (Sergeev et al., 1990; Pulkkinen et al., 1992).

All these theoretical and observational results strongly support the concept of anomalous non-idealness due to microscopic (current-driven) tubulence. Following this line, anomalous resistivities are derived for several specific instabilities in the literature, e.g. the cross-tail instability (Lui et al., 1991) or lower hybrid drift waves (Cattell and Mozer, 1987). However, there is still a significant lack in self-consistent derivation of the general non-ideal consequences of microscopic fluctuations in the ideal collisionfree magnetospheric plasma. Because of that lack, MHD-simulations of magnetospheric dynamics (e.g. Scholer, 1987; Hautz and Scholer, 1987; Otto et al., 1990; Hesse and Birn, 1992; Ugai, 1992; Büchner and Otto, 1993; Hesse et al., 1994) have to use resistivity models which are plausible, but only based on ad hoc assumptions.

In the second part of our paper, we present a new approach to macroscopic non-idealness. In this ap- proach it is not necessary to specify a particular microinstability. It allows us to calculate the non-ideal macroscopic consequences of general fluctuations proceeding on microscopic scales. This approach will help to come to a deeper understanding of the consequences of microscopic collective fluctuations in the Earth's plasma sheet, especially with respect to onset and nonlinear evolution of magnetic reconnection and to derive more realistic resistivity models as input for future MHD-simulations.

\section{Thin current sheets in the near-Earth plasma sheet}

\subsection{The numerical model}

We study the problem of the formation of thin current sheets in the ideal near-Earth plasma sheet by the help of 2- and 3-dimensional MHD simulations. The start configuration in our simulations is based on a 2-dimensional magnetospheric equilibrium including the Earth's dipole field explicitly, which has originally been developed by Fuchs and Voigt (1979). It is a solution of the following inhomogeneous form of the Grad-Shafranov equation

$\Delta A+\mu_{0} \frac{d P}{d A}=M_{D} \frac{\partial}{\partial x} \delta(x) \delta(z)$

with the right hand side representing the singular current distribution of the Earth's dipole. The coordinates $-x, z$ are GSM (Geo Solar Magnetospheric) coordinates. $A, P, M_{D}$ denote the flux function $A(x, z)$, the pressure function $P(A)$, and the dipole moment, respectively. With the ansatz

$P(A)=\frac{k^{2}}{2 \mu_{0}} A^{2}$

(the free parameter $k$ specifies the plasma pressure) the inhomogeneous Grad-Shafranov Eq. (1) becomes linear in $A$ and can be solved by a series of eigenfunctions

$$
\begin{gathered}
A(x, z)=-\frac{M_{D}}{2} \sum_{n} \cos \left(\alpha_{n} z\right) *\left[\operatorname{sign}(x) \exp \left(-\left|\lambda_{n} x\right|\right)\right. \\
\left.-\exp \left(-\left|\lambda_{n}(2 b-x)\right|\right)\right]
\end{gathered}
$$

with eigenvalues $\alpha_{n}=\pi(2 n-1) / 2$ and $\lambda_{n}=\left(\left|\alpha_{n}^{2}-k^{2}\right|\right)^{0.5}$ and $b$ denoting the location of the dayside magnetopause. In our simulations we consider lowest order eigenfunctions, only.

This approach considers the Earth's dipole explicitly and yields a suitable description of the near-Earth plasma sheet (Wiechen et al., 1995, 1996).

Starting with an equilibrium of the type mentioned above, we solve the following set of MHD equations

$$
\begin{aligned}
& \frac{\partial \rho}{\partial t}=-\nabla \cdot(\rho \mathbf{v}) \\
& \frac{\partial \rho \mathbf{v}}{\partial t}=-\nabla \cdot\left(\rho \mathbf{v v}+\frac{1}{2}\left(p+\mathbf{b}^{2}\right) \underline{\mathbf{1}}-\mathbf{b b}\right)
\end{aligned}
$$




$$
\begin{aligned}
& \frac{\partial \mathbf{b}}{\partial t}=\nabla \times(\mathbf{v} \times \mathbf{b}-\eta \mathbf{j}) \\
& \frac{\partial h}{\partial t}=-\nabla \cdot(h \mathbf{v})+\frac{\gamma-1}{\gamma} h^{1-\gamma} \eta \mathbf{j}^{2}
\end{aligned}
$$

$\mathbf{j}=\nabla \times \mathbf{b}$

with $h=(p / 2)^{1 / \gamma}$ and $\rho, p, \mathbf{v}, \mathbf{b}, \mathbf{j}, \eta$, and $\gamma$ denoting the plasma density, pressure, velocity, magnetic field, current density, resistivity and the ratio of specific heats, respectively (here: $\gamma=5 / 3$ ). All quantities are normalized to typical values of the system. Length scales are measured in units of half-thickness $L_{0}$ of plasma sheet, the density is normalized to a typical value $\rho_{0}$ in the center of the plasma sheet and the magnetic field is normalized to a typical value $B_{0}$ at the plasma sheet boundary. Further normalizations (e.g. the Alfvénic transit time) follow in a generic way.

The code we use in our simulations has been developed in its original form by Otto (1987). The 2-dimensional simulations are done in a box with $0 \leq x \leq 10$ and $0 \leq z \leq 1$. For our 3-dimensional simulations the box is extended into $y$-direction with $-5 \leq y \leq 5$. To exclude the dipole singularity from the calculations it is shifted to some $x_{d}<0$ outside the simulation box. The earthward boundary of the simulation box can be interpreted to be located about $5 R_{E}$ tailwards of Earth.

At the earthward boundary of the simulation box we assume a closed ionosphere with $\mathbf{v}=0$. We consider open boundary conditions at the tailward edge. At the boundaries in $y$ and $z$ we have zero normal components of the velocity. The magnetic field $\left(B_{x}, B_{z}\right)$ is symmetric at the boundaries $y_{\min }$ and $y_{\max }$. The whole configuration is assumed to be symmetric with respect to $z=0$.

To study the formation of thin current sheets in the ideal near-Earth plasma sheet due to dissipation-free boundary perturbations we assume localized inflow through the plasma sheet boundary at $z=1$ corresponding to some external electric field. The details of the perturbations are discussed for each example separately.

In both examples we will discuss below we assume a low homogeneous background resistivity corresponding to a magnetic Reynolds number of $R_{M}=1400$. This background resistivity has to be chosen for numerical reasons. It is high as compared with real magnetospheric resistivity which, however, is practically not realizable in MHD simulations. But for our purposes, it is sufficiently low to study a nearly ideal dynamics on the time scales of interest.

\subsection{Three-dimensional simulations of current sheet formation}

Previous ideal MHD simulations (Wiechen and Ziegler, 1998) have shown the formation of thin current sheets in a 2-dimensional near-Earth plasma sheet configuration due to inflow through the plasma sheet boundary. Here we present results of 3-dimensional simulations. In this case, the ideal plasma in the near-Earth plasma sheet has more degrees of freedom to react on boundary perturbations. Thus, it is not clear from the previous 2-dimensional results, whether or not current sheets will form in the course of a 3-dimensional dynamics.

As boundary perturbations, we assume localized inflow through the plasma sheet boundary at $z=1$ concentrated near $x=1$ and $y=0$, i.e.

$\delta v_{z} \sim e^{-200(1-z)^{2}-0.1 y^{2}} \sin \left(\pi\left(1-e^{-x \ln 2}\right)\right)$

The amplitude of the perturbation corresponds to an external electric field of $E_{\text {ext }} \approx 0.01$ which is similar to that in previous studies of the influence of external electric fields on magnetotail reconnection (Birn and Schindler, 1986).

Figure 1 shows a surface plot of the current density at $y=0$ after $10 \tau_{A}$. One finds signatures of the perturbation, but the current density is still rather smooth and quite similar to the initial unperturbated profile. This, however, changes drastically looking at a snapshot after $70 \tau_{A}$ (Fig. 2). At this time one finds a significant thin current sheet developing in the center of the plasma sheet (with a maximum in the midnight-meridional plane typically extended in $y$ like the inflow) which is the consequence of the perturbation of the plasma sheet boundary. These results show that the formation of thin current sheets has to be interpreted as a general consequence of dissipation-free boundary perturbations in an ideal plasma, even in the case of a 3-dimensional dynamics.

\subsection{The location of thin current sheets}

A question of special interest in context with the formation of thin current sheets in the central plasma sheet is that of their location. Observations show thin current sheets in the late growth phase predomenantly in the near-Earth region of the central plasma sheet about 8-10 $R_{E}$ tailwards of Earth (Sergeev et al., 1990; Pulkkinen et al., 1992). In our present paper we study this problem with the help of 2-dimensional MHD-

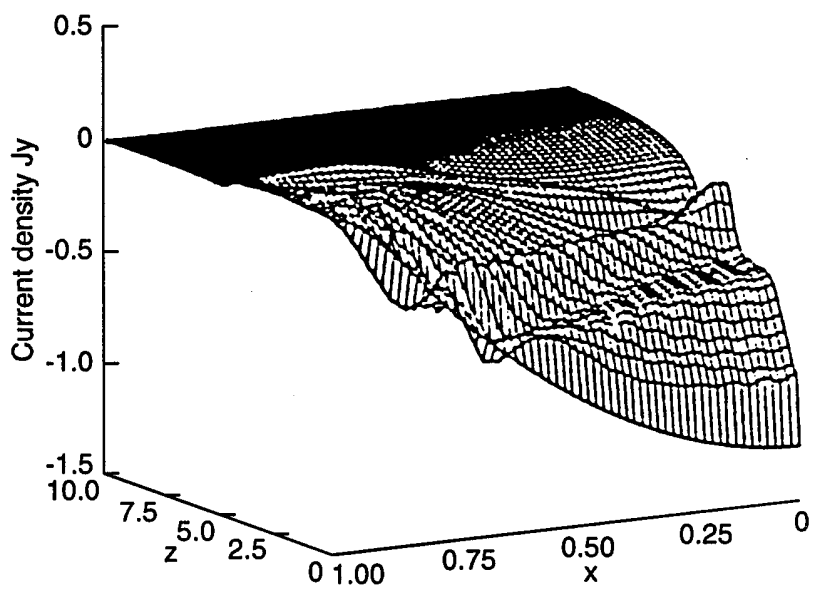

Fig. 1. 3-dimensional simulation of current sheet formation. Surface plot of the current density at $y=0$ being still almost smooth after $10 \tau_{A}$ 


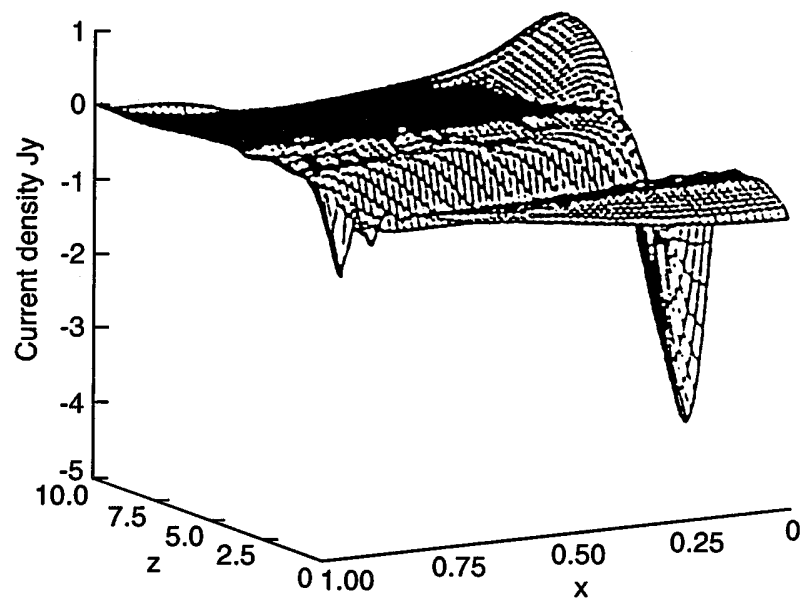

Fig. 2. 3-dimensional simulation of current sheet formation. Surface plot of the current density at $y=0$ after $70 \tau_{A}$ showing a developing thin current sheet in the central plasma sheet due to local boundary perturbations

simulations of the near-Earth plasma sheet, again using a start configuration of the type mentioned in sect. 2.1. As in the previous 3-dimensional example we assume a boundary perturbation due to local inflow through the northern plasma sheet boundary at $z=1$ corresponding to an external electric field of $E_{\text {ext }} \approx 0.01$.

But now, we vary the location of the inflow with respect to $x$, i.e. in tailward direction, and discuss three examples. In the first case, the inflow is concentrated near to $x_{E}=2.1$ (case 1), in the second case near to $x_{E}=4.2$ (case 2), and in the third case near to $x_{E}=6.3$ (case 3), respectively (see Fig. 3). Figure 4 shows snapshots of the time evolution of the corresponding current densities for all three examples. The snapshots on the left side show the current densities in early phases of the dynamics, i.e. after $5 \tau_{A}$ for case 1 , after $7.5 \tau_{A}$ for case 2 and after $10 \tau_{A}$ for case 3 . For all three cases the current density is still widely unaffected by the perturbation at these early times.

In the course of time, however, thin current sheets develop in the central plasma sheet. This can be seen in the snapshots on the right side of Fig. 4 showing the current densities after $45 \tau_{A}$ (case 1), 67.5 $\tau_{A}$ (case 2), and $90 \tau_{A}$ (case 3), respectively. The time scales of current sheet formation increase with increasing distance of the localized boundary perturbation from Earth. The location of the current sheets, however, turns out to be widely independent from the localization of the boundary perturbation. In all three cases thin current sheets develop at about $x=3$ cooresponding to about $10 R_{E}$ tailwards of Earth.

Thus, both observations and simulations being in excellent agreement indicate that this location is a particular one with respect to the formation of current sheets in the plasma sheet.

Our results show that it is not necessary to assume perturbations at the earthward boundary of the plasma sheet as assumed in a scenario discussed by Schindler and Birn (1993) and Schindler (1994). Even perturbations at the nothern (and southern) plasma sheet

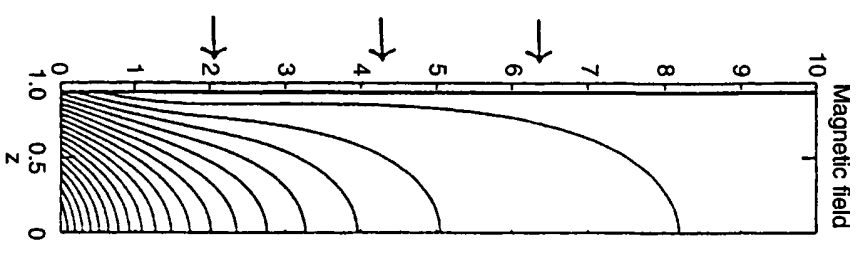

Fig. 3. 2-dimensional simulation of current sheet formation. Unperturbated magnetic field of the near-Earth plasma sheet at $t=0$. Boundary perturbations due to localized inflow through the plasma sheet boundary at $z=1$ are switched on near $x_{E}=2.1$ (case 1), $x_{E}=4.2$ (case 2) and $x_{E}=6.3$ (case 3)

boundary yield current sheets located at $\approx 8-10 R_{E}$ widely independent from the localization of the perturbation.

This result can be interpreted as a consequence of the fact that the variations with respect to $x$ of all plasma parameters are significantly stronger in the near-Earth plasma sheet with a significant dipole contribution as compared with a tail configuration considered e.g. by Schindler and Birn (1993) and Schindler (1994). Thus, current sheets form where the perturbation acts most effectively (i.e. near the earthward edge where the density is high) and not where the amplitude is maximal but the density decreased significantly. Besides, the location of the current sheets will be influenced by the closed ionospheric boundary.

\section{Macroscopic non-idealness due to microscopic fluctuations}

The results of our MHD simulations strongly support the concept of magnetic reconnection enabled by macroscopic non-idealness due to microscopic fluctuations. Now we want to discuss how the macroscopic non-ideal consequences of microscopic collective fluctuations can be determined quantitatively in a selfconsistent way. To do this we have to pass over to a kinetic description and consider a plasma with a dynamics governed by the Vlasov equations

$\frac{\partial f_{s}}{\partial t}+\mathbf{v} \cdot \nabla f_{s}+\frac{e_{s}}{m_{s}}\left(\mathbf{E}+\frac{1}{c} \mathbf{v} \times \mathbf{B}\right) \cdot \frac{\partial f_{s}}{\partial \mathbf{v}}=0$

together with Maxwell's equations $\left[f_{s}, e_{s}, m_{s}\right.$ denote distribution functions in 6-dimensional phase space, charge and mass of electrons $(s=e)$ and ions $(s=i)$, respectively]. The final aim is to calculate Ohm's law

$\mathbf{E}+\mathbf{v} \times \mathbf{B}=\mathbf{R}$

with a macroscopic non-ideal term

$\mathbf{R}=\left.\sum_{s} e_{s} n_{s} \int \mathbf{v} \frac{\partial f_{s}}{\partial t}\right|_{\text {macro }} \mathrm{d}^{3} v$

resulting not from collisions but from collective fluctuations ( $n_{s}$ denotes the number density of electrons and ions, respectively). 

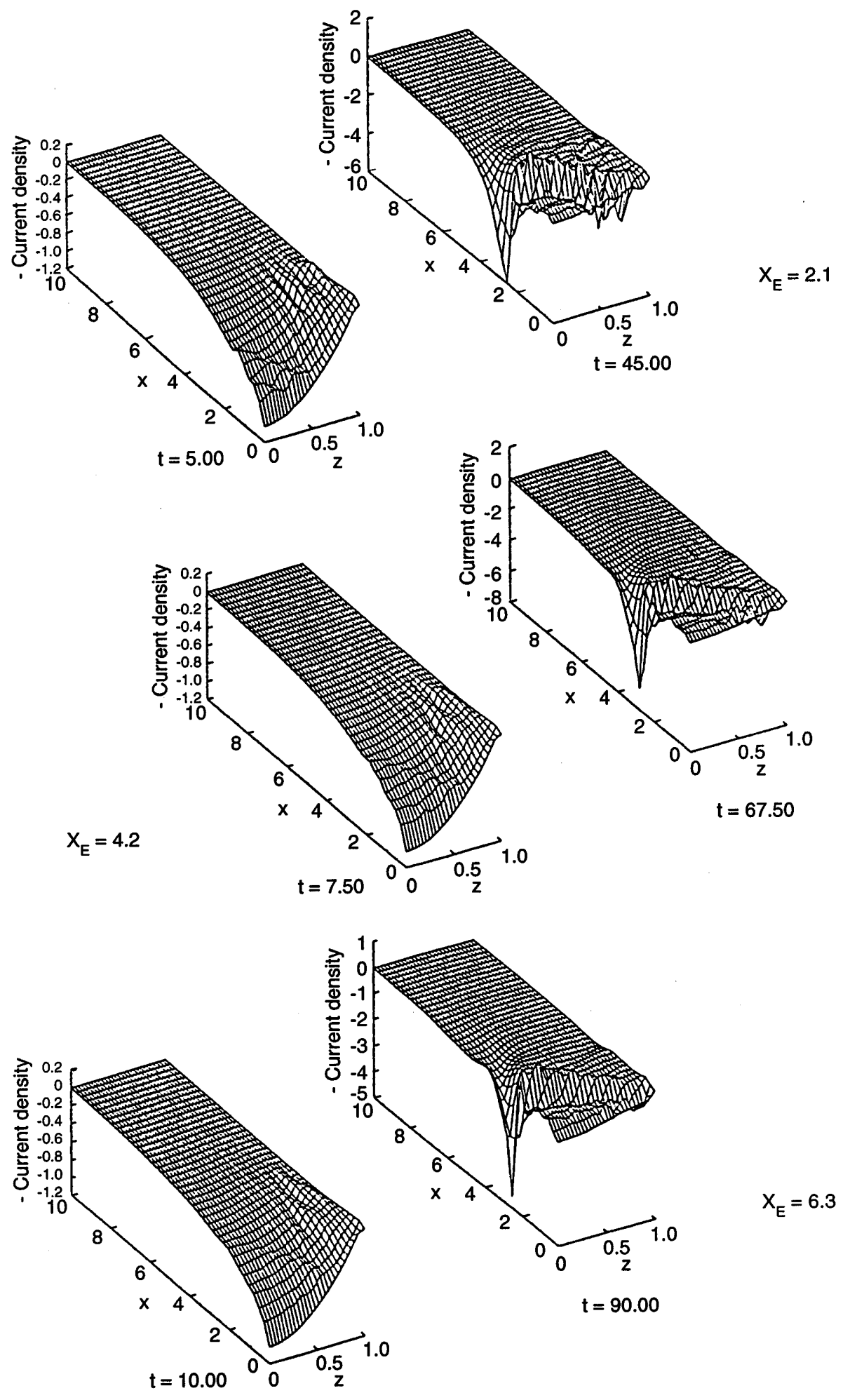

Fig. 4. 2-dimensional simulation of current sheet formation. Surface plots of the current densities for the cases $1-3$ in an early phase of the dynamics (after $5 \tau_{A}$ (case 1), 7.5 $\tau_{A}$ (case 2) and 10 $\tau_{A}$ (case 3)) and in a later phase (after $45 \tau_{A}$ (case 1), $67.7 \tau_{A}$ (case 2) and $90 \tau_{A}$ (case 3 )). In all three cases thin current sheets develop near $x=3$ corresponding to about 8-10 $R_{E}$ tailwards of Earth

In the following, we will show that this can be realized by a new approach to anomalous non-idealness which has been developed originally in the framework of a theory for the relaxation of collisionless matter (e.g. Wiechen et al., 1988; Ziegler and Wiechen, 1989; Wiechen and Ziegler, 1994). This approach allows us to calculate anomalous non-idealness as the macroscopic consequence of general microscopic collective fluctuations, i.e. we do not have to take into account details of specific microscopic instabilities, but only a fundamental consequence of microscopic turbulence.

The basic mechanism under consideration that yields non-idealness on macroscopic scales in a collisionfree plasma is mixing in 6-dimensional phase space, i.e. we 
assume a dynamics with (perturbated) distribution functions fluctuating on smaller and smaller scales. In the course of time, these more and more microscopic fluctuations will not be resolved anymore from a macroscopic point of view. Thus, on macroscopic scales the distribution functions become stationary. The transition from the exact collisionfree microscopic dynamics to distribution functions on macroscopic, averaged scales yields an increase of entropy corresponding to some non-idealness. This non-idealness can be described in terms of violation of phase-space density conservation. Figure 5 illustrates this by the help of a simple schematic picture.

Let us assume a simple distribution function $f(\mathbf{r}, \mathbf{v})$ taking some value $\eta>0$ in some domain in phase space with volume $\phi_{\eta}$ and being 0 else. Now, due to some perturbation or instability, the distribution function starts to filamentate with fluctuations taking place on smaller and smaller scales. In the framework of the exact collisionfree dynamics, for all times the distribution function will take only one value, i.e. $\eta$, and the total volume occupied by the filamentated distribution function will be $\phi_{\eta}$ independent from how this volume is spread out in phase space. This conservation of phase-space density conservation can be described by a so called reduced distribution function $g(\phi)$ (e.g. Tremaine et al., 1986) defined as the inverse function of

$\phi(g)=\int \Theta[f(\mathbf{r}, \mathbf{v})-g] \mathrm{d}^{3} r \mathrm{~d}^{3} v$

denoting the phase-space volume with $f \geq g(\Theta(x)$ is the unit step function with $\Theta(x)=1$ for $x \geq 1$ and 0 else). In our present simple example $g(\phi)$ contains just the information that the distribution function is $\eta$ in an overall volume $\phi_{\eta}$. This reduced distribution function is a constant of motion in the exact microscopic dynamics.

For filamentations proceeding on more and more microscopic scales these microscopic fluctuations will not be resolved anymore on macroscopic scales yielding averaged (coarse-grained) distribution function $\bar{f}(\mathbf{r}, \mathbf{v})$. In our example (see Fig. 6), this microscopic distribution function will have a value $\bar{\eta}<\eta$ occupying a phasespace volume $\bar{\phi}>\phi_{\eta}$ corresponding to a violation of phase space density conservation. As a consequence, the reduced distribution function $\bar{g}(\phi)$ corresponding to the macroscopic distribution in phase space, i.e. the inverse function of

$\phi(g)=\int \Theta[\bar{f}(\mathbf{r}, \mathbf{v})-g] \mathrm{d}^{3} r \mathrm{~d}^{3} v$

is different from the reduced distribution function corresponding to the exact microscopic distribution function as schematically shown.

The crucial idea in our approach is to calculate the macroscopic non-ideal consequences of microscopic fluctuations by the help of reduced distribution functions without to follow the complicated dynamics in phase space.
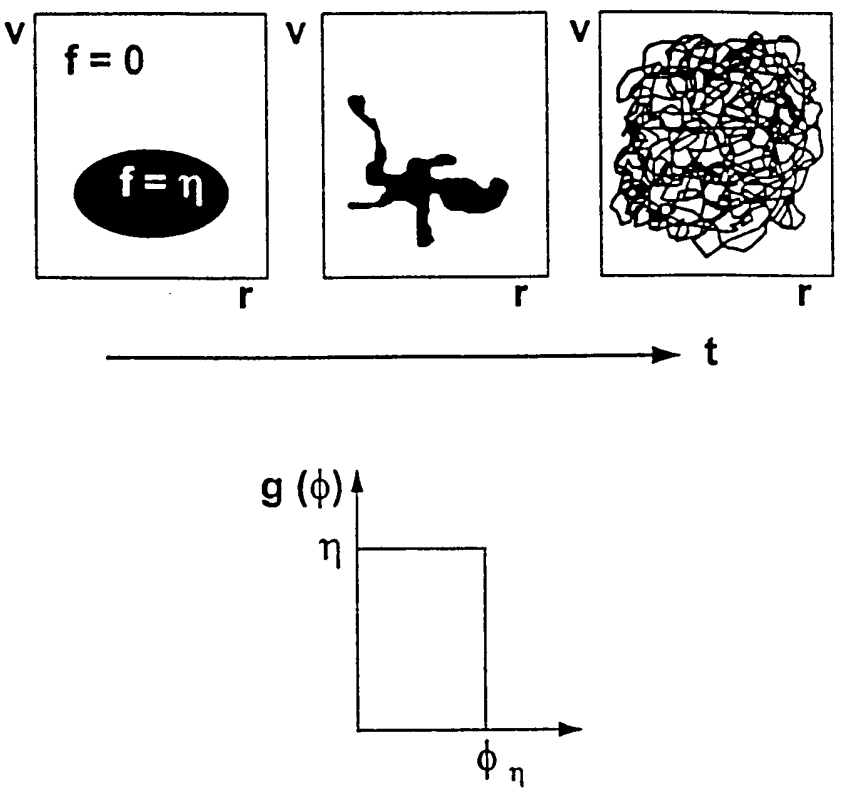

Fig. 5. Sketch of a simple distribution function with some value $\eta$ in some domain in phase space of total volume $\phi_{\eta}$ filamentating in phase space (upper row) together with the corresponding reduced distribution function describing phase-space density conservation in the framework of the exact microscopic dynamics

Before going into technical details we want to motivate our approach by the help of a simple example. Dropping a drop of ink into some water one can determine the final state (some light blue liquid) without calculating the dynamics of the molecules by the help of three input parameters and one basic assumption. First one must know the volume of the drop, second the darkness of the ink and third the ratio between the volume of ink and the volume of water. Together with the basic assumption of homogeneous mixing between ink and water this knowledge is sufficient to determine the final state quantitatively.

In our approach we proceed to some extent by analogy. We start with unperturbated distribution functions $f_{s}(\mathbf{r}, \mathbf{v})$ of electrons and ions, respectively and calculate the corresponding reduced distribution functions $g_{s}(\phi)$. (Considering our simple 1-value distribution in Fig. 5 and our ink-water example, $\eta$ would correspond to the darkness of the ink and $\phi$ to the volume of the drop.)

The first main question we have to answer now is how the reduced distribution functions are transformed in principle by the transition to macroscopic scales? This question can be answered taking into account two strict constraints. The first constraint is that we have to describe the coarse-graining (averaging) of a distribution function in phase space. The second constraint is that we have filamentation due to collective fluctuations and no collisions. Considering these two constraints together with the further assumption of strong, homogeneous filamentation one can show (e.g. Ziegler and Wiechen, 1989) that the reduced distribution functions correspond- 

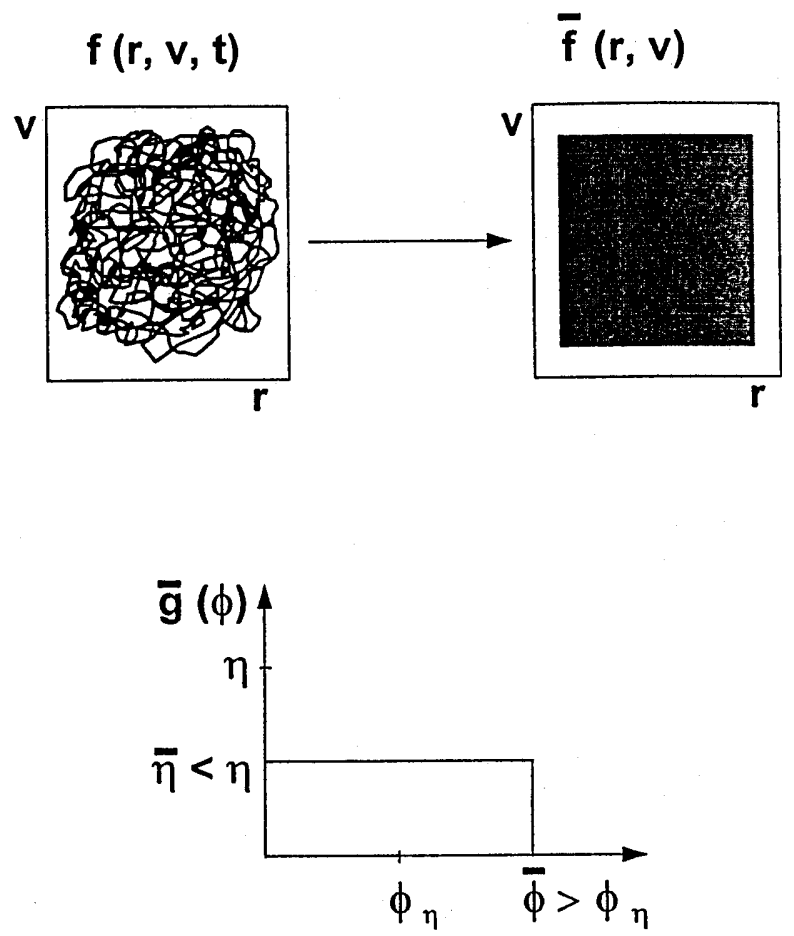

Fig. 6. Sketch of a simple distribution function filamentating in phase space (left) and the corresponding coarse-grained distribution function on macroscopic scales (right). Due to the averaging the value of the distribution function is decreased, the occupied volume increased. The consequences for the corresponding reduced distribution function is schematically shown at the bottom

ing to coarse-grained macroscopic distributions in phase space can be calculated by a self-similar transformation

$\bar{g}_{s}(\phi)=\frac{1}{\alpha} g_{s}\left(\frac{\phi}{\alpha}\right)$ with $\alpha \geq 1$

The parameter $\alpha$ can be interpreted as a measure for the macroscopic scale on which the microscopic fluctuations are not resolved anymore. (In this coarse-graining model we do not consider further restrictions on the dynamics in configuration space as it was done in some former formulation by Wiechen and Ziegler, 1994.)

The second main question is to determine the parameter $\alpha$ quantitatively. We know that the perturbated filamentating system is not in equilibrium, otherwise there would be no filamentation. Thus some part of the energy of the perturbated system must be inherent in the microscopic fluctuations. This amount of energy $E_{f l u c}$ can be calculated as the difference between the energy of the perturbated system $E_{\text {syst }}$ and the minimum energy of all distributions corresponding to the original reduced distribution functions $g_{s}(\phi)$.

$E_{f l u c}=E_{s y s t}-\min \left(\left.E\right|_{g_{s}}\right)$

The minimum energy state with $E=\min \left(\left.E\right|_{g_{s}}\right)$ corresponds to the (globally) stable equilibrium principally accessible in the framework of the collisionfree microscopic dynamics and can be calculated by a suitable energy minimizing techniques (for details see e.g.
Schindler and Goldstein, 1983; Wiechen and Ziegler, 1994, 1998). Knowing the amount of energy $E_{\text {fluc }}$ inherent in the microscopic fluctuations, the parameter $\alpha$ can be calculated explicitly as a function of $E_{f l u c}$, i.e. $\hat{\alpha}=\alpha\left(E_{f l u c}\right)$ (e.g. Ziegler and Wiechen, 1989; Wiechen and Ziegler, 1994). (Considering our simple example of a drop of ink in some water, $\hat{\alpha}$ corresponds in some sense to the ratio of the volume of the ink and the volume of the water.)

Thus we can calculate the reduced distribution functions corresponding to the coarse-grained, macroscopic distributions in phase space for electrons and ions, respectively, and know the non-ideal consequences of microscopic fluctuations in terms of violation of phase space density conservation on macroscopic scales quantitatively.

Now we have to answer the third fundamental question, i.e. we have to determine the coarse-grained macroscopic phase-space distribution functions itself. Obviously, there is an infinite number of phase-space distributions to each reduced distribution function. Thus we need a selection criterion. We know that the macroscpic phase space distributions show no microscopic fluctuations. Thus we can assume them to be stationary states, at least on the time scales of interest. With this assumption the phase-space distributions can be calculated as stationary points of energy, i.e. as the equilibrium distribution functions $\bar{f}_{s}(\mathbf{r}, \mathbf{v})$ corresponding to $\hat{\alpha}^{-1} g_{s}\left(\phi \hat{\alpha}^{-1}\right)$. This can be done using an appropriate variational analysis techniques. Without going into details we note that this macroscopic stationary state has just the energy $E_{s y s t}$ of the perturbated system because $\alpha\left(E_{f l u c}\right)=\alpha\left(E_{s y s t}-\min \left(\left.E\right|_{g_{s}}\right)\right.$ depends implicitly on $E_{s y s t}$. For a detailed discussion of this point and the energy minimizing techniques we refer to Ziegler and Wiechen 1989; and Wiechen and Ziegler 1994, 1998. The uniquness of this equilibrium for a specific example is not strictly clear, however, practically one will find only one as studies of this problem have shown (e.g. Ziegler and Wiechen, 1989; Wiechen and Ziegler, 1993).

Thus, we can explicitly calculate the macroscopic, (in 6-dimensional phase space with respect to $\mathbf{r}$ and $\mathbf{v}$ ) coarse-grained distribution functions $\bar{f}_{s}(\mathbf{r}, \mathbf{v})$ corresponding to strongly filamentating microscopic distributions. Now we can do the final step and determine the macroscopic non-idealness $\mathbf{R}$ quantitatively as the consequence of microscopic fluctuations. To do this we make the ansatz

$\mathbf{R}=\sum_{s} e_{s} n_{s} \int \mathbf{v} \frac{f_{s}(\mathbf{r}, \mathbf{v})-\bar{f}_{s}(\mathbf{r}, \mathbf{v})}{\tau} \mathrm{d}^{3} v$

$f_{s}$ denotes the electron and ion distribution functions of the unperturbated system, $\bar{f}_{s}$ denotes the coarsegrained electron and ion distributions resulting from our approach and $\tau$ denotes some typical time scale of the collective fluctuations. This time scale is a priori unknown, it has to be derived from more detailed theoretical studies or from in situ measurements in the plasma sheet. The ansatz Eq. (17) for the non-idealness is formally in plausible analogy to the Krook model for resistivity (Krall and Trivelpiece, 1986) but now it 
describing the non-ideal macroscopic consequences of collective microscopic fluctuations and not the consequences of collisions. Details of the specific form of the macroscopic non-idealness have to be studied further.

\section{Summary and discussion}

In our paper we discussed new aspects, both in MHD and kinetic theory, of plasma sheet dynamics considering the problem of anomalous non-idealness necessary for magnetic reconnection to occur in the, in general, highly ideal (in the framework of MHD) or collisionfree (from a kinetic point of view) magnetospheric plasma.

In the first part we studied MHD simulations of current sheet formation in the near-Earth plasma sheet including the Earth's dipole field explicitly. Thus, the study of typical near-Earth features is not restricted to suitable assumptions for earthward boundary conditions, rather the dynamics of the near-Earth plasma and field itself is simulated.

We have shown that perturbations due to local inflow through the northern (or southern) boundary of the plasma sheet lead to the formation of thin current sheets in the near-Earth region without any perturbations at the earthward boundary. The location of the current sheet formation turnout to be widely independent from the location of the boundary perturbation. In all examples we find developing thin current sheets about 8-10 $R_{E}$ tailwards of Earth in agreement with observations. These results support the concept of macroscopic anomalous non-idealness due to (current-driven) microinstabilities.

In the second part of our paper we discussed the problem of how to calculate quantitatively the macroscopic non-ideal consequences of microscopic fluctuations in a self-consistent way. We presented a new approach to this problem which does not demand details of specific microscopic instabilities. We rather consider a general feature of microscopic turbulence, i.e. filamentation in phase space. In this approach we follow the idea of calculating the non-ideal consequences due to violation of phase-space density conservation on macroscopic scales assuming fluctuations proceeding on more and more microscopic scales.

Assuming further that this process yields coarsegrained distribution functions which are stationary, at least on the time scales of interest, one can calculate the macroscopic distribution functions $\bar{f}_{s}(\mathbf{r}, \mathbf{v})$ for electrons and ions explicitly from the knowledge to which extent the phase-space density conservation is violated on macroscopic scales. In a final step we calculate the corresponding macroscopic non-idealness by the help of the Krook model for resistivity, which now describes the anomalous non-ideal consequences of microscopic collective fluctuations.

This approach is new in the present context and there are still open questions. This refers to the problem of a suitable typical time scale which must be known in our model resistivity. A second problem is how the filamentation can be described as a local process in the plasma sheet enviroment (i.e. one needs some proper boundary conditions). These questions have to be studied in more detail in the future.

Although we discuss our model only qualitatively in the present paper without an explicit quantitative example, we emphasize that our model, being part of a theory for the relaxation of collisionless matter, has been successfully realized in that relaxation context (e.g. Ziegler and Wiechen, 1989; Wiechen and Ziegler, 1993, 1994; Stahl et al., 1998). Thus we expect that our model will help to improve the understanding of the coupling between microscopic and macroscopic processes in the Earth's plasma sheet.

Acknowledgement. The author wishes to thank both referees for helpful comments.

Topical Editor K.-H. Glassmeier thanks G. Hornig and A. Otto for their help in evaluating this paper.

\section{References}

Birn, J., and K. Schindler, On the Influence of an external electric field on magnetotail reconnection, J. Geophys. Res., 91, 8817, 1986.

Büchner, J., and A. Otto, Consequences of chaotic particle motion for the stability of space plasma current sheets, in Proceedings of the 4th International Conference on Plasma Physics and Controlled Nuclear Fusion, Eur. Space Agency Spec. Publ., ESA SP-351, 105, 1993.

Büchner, J., and L. M. Zelenyi, Regular and chaotic charged particle motion in magnetotail-like field reversals, J. Geophys. Res., 94, 11,821, 1989.

Cattell, C. A., and F. S. Mozer, Substorm-associated lower hybrid waves in the plasma sheet observed by ISEE-1, in A. T. Y. Lui (ed.) Magnetotail Physics Johns Hopkins University Press, Baltimore, 119, 1987.

Fuchs, K., and G.-H. Voigt, Self-consistent theory of a magnetospheric B-field model, in vol. 21 W. P. Olsen (ed.) Quantitative Modeling of Magnetospheric Processes, Geophys. Monogr. Ser., AGU, Washington, D.C., 86, 1979.

Haerendel, G., Microscopic processes related to reconnection, J. Atmos. Terr. Phys., 40, 343, 1978.

Hahm, T. S., and R. M. Kulsrud, Forced magnetical reconnection, Phys. Scr., 2, 525, 1982.

Hautz, R., and M. Scholer, Numerical simulations on the structure of plasmoids in the deep tail, Geophys. Res. Lett., 14, 969, 1987.

Hesse, M., and J. Birn, Three-dimensional MHD modeling of magnetotail dynamics for different polytropic indices, J. Geophys. Res., 97, 3965, 1992.

Hesse, M., J. Birn, D. N. Baker, and T. I. Pulkkinen, MHD simulations of substorm dynamics including an inner magnetotail, J. R. Kan J. D. Craven, S.-I. Akasofu (ed.) in Proceedings of the 2nd International Conference on Substorms, University of Alaska Fairbanks, 493, 1994.

Huba, J. D., N. T. Gladd, and K. Papadopoulos, The lower-hybrid drift instability as a source of anomalous resistivity for magnetic field line reconnection, Geophys. Res. Lett., 4, 125, 1977.

Huba, J. D., N. T. Gladd, and K. Papadopoulos, Lower-hybrid drift wave turbulence in the distant magnetotail, J. Geophys. Res., 83, 5217, 1978.

Krall, N. A., and A. W. Trivelpiece, Principles of Plasma Physics, San Francisco Press, San Francisco, 316, 1986.

Lui, A. T. Y., Y. C. L. Chang, A. Mankofsky, H. K. Wong, and D. Winske, A cross-field current instability for substorm expansions, J. Geophys. Res., 96, 11,389, 1991. 
Lui, A. T. Y., P. H. Yoon, and C.-L. Chang, Quasi-linear analysis of ion Weibel instability in the earth's neutral sheet, J. Geophys. Res., 98, 153, 1993.

Moldwin, M. B., and W. J. Hughes, Geomagnetic substorm association of substorms, J. Geophys. Res., 98, 81, 1993.

Otto, A., Zur linearen und nichtlinearen Analyse resistiver Instabilitätsprozesse in schwach zweidimensionalen Gleichgewichten (Ph.D. Thesis), Ruhr-Univ. Bochum, Germany, 1987.

Otto, A., K. Schindler, and J. Birn, Quantitative study of the nonlinear formation and acceleration of plasmoids in the Earth's magnetotail, J. Geophys. Res., 95, 15,023, 1990.

Papadopoulos, K, The role of microturbulence on collisionless reconnection in S.-I. Akasofu (ed.) Dynamics of the Magnetosphere, Reidel, 289, 1979.

Parker, E. N., Topological dissipation and the small-scale fields in turbulent gases, Astrophys. J., 174, 499, 1972.

Pulkkinen, T. I., D. N. Baker, R. J. Pellinen, J. Büchner, H. E. J. Koskinen, R. E. Lopez, R. L. Dyson, and L. A. Frank, Particle scattering and current sheet stability in the geomagnetic tail during the substorm growth phase, J. Geophys. Res., 97, 19,283, 1992.

Rastätter, L., A. Voge, and L. Schindler, On current sheets in twodimensional ideal magnetohydrodynamics caused by pressure perturbations, Phys. Plasmas, 1, 3414, 1994.

Schindler, K., A theory of substorm mechanism, J. Geophys. Res., 79, 2803, 1974.

Schindler, K., and H. Goldstein, A nonlinear kinetic energy principle for two-dimensional collision-free plasmas, Phys. Fluids, 26, 2222, 1983.

Schindler, K., and J. Birn, On the cause of thin current sheets in the near-Earth magnetotail and their possible significance for magnetospheric substorms, J. Geophys. Res., 98, 15,477, 1993.

Schindler, K., Aspects of magnetospheric activity and parallel electric fields, Phys. Scr., T50, 20, 1994.

Scholer, M., Earthward plasma flow during near-Earth magnetic reconnection: numerical simulations, J. Geophys. Res., 92, 12,425, 1987.

Sergeev, V. A., P. Tanskanen, K. Mursula, A. Korth, and R. C. Elphic, Current sheet thickness in the near-Earth plasma sheet during dubstorm growth phase, J. Geophys. Res., 95, 3819, 1990.

Stahl, B., H. J. Ziegler, H. Wiechen, and A. Schröer, A quantitative model for relaxation of rotationally invariant collisionless selfgravitating systems, Phys Scr., T74, 93, 1998.

Tremaine, S., M. Hénon, and D. Lynden-Bell, H-functions and mixing in violant relaxation, Mon. Not. R. Astrom Soc., 219, $285,1986$.

Ugai, M., Computer studies on development of the fast reconnection mechanism for different resistivity models, Phys. Fluids B, 4, 2953, 1992.

Voge, A., A. Otto, and K. Schindler, Nonlinear current-sheet formation in ideal plasmas, J. Geophys. Res., 99, 21,241, 1994.

Wiechen, H., H. J. Ziegler, and K. Schindler, Relaxation of collisionless self-gravitating matter, Mon. Not. R. astrom Soc., 232, 632, 1988.

Wiechen, H., and H. J. Ziegler, Globally stable equilibria of collisionless self-gravitating matter with non-vanishing total angular momentum, Astrophys. J., 408, 293, 1993.

Wiechen, H., and H. J. Ziegler, Relaxation of collisionless plasmas, J. Plasma Physics, 51, 341, 1994.

Wiechen, H., J. Büchner, and A. Otto, Reconnection in a dipoledominated magnetosphere - a two-Dimensional model, J. Geophys. Res., 100, 19,259, 1995.

Wiechen, H., J. Büchner, and A. Otto, Reconnection in the nearEarth plasma sheet - a three-dimensional model, J. Geophys. Res., 101, 24,911, 1996.

Wiechen, H., J. Büchner, and A. Otto, Driven reconnection in the near-Earth plasma sheet, Adv. Space Res., 19, 1939, 1997.

Wiechen, H., and H. J. Ziegler, Magnetic reconnection: on new aspects of the microscopic cause of localized dissipation, Phys. Scr., T74, 14, 1998.

Wiegelmann, T., and K. Schindler, Formation of thin current sheets in a quasistatic magnetotail model, Geophys. Res. Lett., 15, 2057, 1995.

Ziegler, H. J., and H. Wiechen, Relaxation of collisionless selfgravitating matter: the final state, Mon. Not. R. Astrom Soc., 238, 1261, 1989. 